\title{
Catalytic features and crystal structure of a tau class glutathione transferase from Glycine max specifically upregulated in response to soybean mosaic virus infections
}

\author{
Katholiki Skopelitou $^{\mathrm{a}, 1}$, Abdi W. Muleta ${ }^{\mathrm{b}, 1}$, Anastassios C. Papageorgiou ${ }^{\mathrm{b}}$, \\ Evangelia Chronopoulou ${ }^{\mathrm{a}}$, Nikolaos E. Labrou ${ }^{\mathrm{a}, *}$

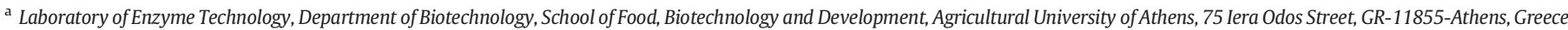 \\ b Turku Centre for Biotechnology, University of Turku and Åbo Akademi University, Turku 20521, Finland
}

\section{A R T I C L E I N F O}

\section{Article history:}

Received 14 August 2014

Received in revised form 9 November 2014

Accepted 21 November 2014

Available online 3 December 2014

\section{Keywords:}

Biotic and abiotic stress

Enzyme catalysis

Herbicide detoxification

Tau class GST

X-ray structure

\begin{abstract}
A B S T R A C T
The plant tau class glutathione transferases (GSTs) play important roles in biotic and abiotic stress tolerance in crops and weeds. In this study, we systematically examined the catalytic and structural features of a GST isoenzyme from Glycine max (GmGSTU10-10). GmGSTU10-10 is a unique isoenzyme in soybean that is specifically expressed in response to biotic stress caused by soybean mosaic virus (SMV) infections. GmGSTU10-10 was cloned, expressed in Escherichia coli, purified and characterized. The results showed that GmGSTU10-10 catalyzes several different reactions and exhibits wide substrate specificity. Of particular importance is the finding that the enzyme shows high antioxidant catalytic function and acts as hydroperoxidase. In addition, its $\mathrm{K}_{\mathrm{m}}$ for GSH is significantly lower, compared to other plant GSTs, suggesting that GmGSTU10-10 is able to perform efficient catalysis under conditions where the concentration of reduced glutathione is low (e.g. oxidative stress). The crystal structure of GmGSTU10-10 was solved by molecular replacement at 1.6 Å resolution in complex with glutathione sulfenic acid (GSOH). Structural analysis showed that GmGSTU10-10 shares the same overall fold and domain organization as other plant cytosolic GSTs; however, major variations were identified in helix H9 and the upper part of helix $\mathrm{H} 4$ that affect the size of the active site pockets, substrate recognition and the catalytic mechanism. The results of the present study provide new information into GST diversity and give further insights into the complex regulation and enzymatic functions of this plant gene superfamily.
\end{abstract}

c) 2014 Elsevier B.V. All rights reserved.

\section{Introduction}

Plant glutathione S-transferases (GSTs; EC 2.5.1.18) are enzymes that catalyze the conjugation of reduced glutathione (GSH; $\gamma$-GluCys-Gly) to electrophilic centers of a wide variety of, mainly hydrophobic, compounds, both endogenous and xenobiotic [1-4]. GSTs are implicated in pesticide detoxification [5-7], in responses to abiotic and biotic stress (infection, heavy metals, UV radiation, etc) [8-11], as well as in hormonal regulation and developmental change [12-16]. GSTs can be found in plants from early embryogenesis to senescence [17].

GSTs comprise a large, complex gene family in plants. Based on a variety of criteria (e.g. sequence relatedness, immunological, kinetic

Abbreviations: CDNB, 1-chloro-2,4-dinitrobenzene; $\mathrm{CuOOH}$, cumene hydroperoxide fluorodifen, 4-nitrophenyl 2-nitro-4-trifluoromethylphenyl ether; GSH, glutathione; $\mathrm{GSOH}$, glutathione sulfenic acid; GST, glutathione transferase; $\mathrm{Nb}$-GSH, $\mathrm{S}$-(p-nitrobenzyl)glutathione; SMV, soybean mosaic virus; t-BuOOH, tert-butyl peroxide

* Corresponding author. Tel./fax: + 302105294308.

E-mail address: lambrou@aua.gr (N.E. Labrou).

1 Joint first authors. and structural properties), plant soluble GSTs can be subdivided to distinct classes: phi $(F)$, tau $(U)$, zeta $(Z)$, theta $(T)$, lambda $(\lambda)$, dehydroascorbate reductase (DHAR), and tetrachlorohydroquinone dehalogenase (TCHQD) $[1,2,12,13]$. The tau class, in particular, is the most abundant of all GST classes and its members play important roles in stress tolerance and secondary metabolism as well as catalyzing the detoxification of herbicides in crops and weeds $[9,13-15$, 17-19].

According to numerous crystallographic studies, GSTs display significant structural conservation $[8,15,16]$. The soluble plant GSTs are homo- and hetero-dimeric enzymes with 23-30 kDa subunits and average length of $200-250$ amino acids $[1,8,12,13,16,20]$. Each subunit consists of two domains, the N-terminal domain with $\alpha / \beta$ topology and the C-terminal domain with $\alpha$-helical structure. Each subunit has a relatively independent active site, composed of the G-site, which is primarily responsible for binding GSH or other closely related peptides (e.g. homoglutathione), and the H-site, which is the site where hydrophobic electrophile substrates bind $[8,16]$. The catalytic residue of GSTs from theta, zeta, phi and tau classes is the amino acid serine $[1,12,13,16]$. The $\mathrm{G}$ - and $\mathrm{H}$-sites are typically formed 
from residues of $\mathrm{N}$ - and $\mathrm{C}$-terminal domains, respectively. Residues of the $\mathrm{H}$-site are not conserved compared to those of the G-site across cytosolic GSTs [1,10-12,16,21]. GSTs, therefore, display wide substrate specificity toward electrophile molecules including organic halides, organic hydroperoxides, epoxides, arene oxides, $\alpha$-and $\beta$ unsaturated carbonyls, organic nitrate esters, and organic thiocyanates $[1,10,11,22,23]$. Selected GSTs from tau, theta and phi classes exhibit peroxidase activity [12-16] by reducing lipid hydroperoxides directly and by removing lipid peroxidation end products like alkenals, 4-hydroxynonenal, and other $\alpha, \beta$-unsaturated aldehydes [3,9-11]. They also display thioltransferase and dehydroascorbate reductase activity $[1,10,12]$. Moreover, they are also involved in GSH-dependent isomerization reactions, the synthesis of sulfurcontaining secondary metabolites, and the conjugation, transport and storage of reactive oxylipins, phenolics and flavonoids [2].

There are 25 GST isoenzymes in soybean (Glycine max) for which their substrate specificity has been studied towards different herbicides (diphenyl ether, chloroacetanilide, sulfonylurea) and xenobiotic substrates $[16,17]$. However, the results of those studies pose the question of why there are so many different GSTs in soybeans with high homologous primary and secondary structures and overlapping substrate specificities. Here we report the characterization and analysis of the structural and functional features of a tau class glutathione transferase (GmGSTU10-10) from G. max. GmGSTU10-10 is differentially over-expressed in response to soybean mosaic virus (SMV) infections. In particular, among the 25 different GST isoenzymes in soybean, GmGSTU10-10 is the sole GST transcript that is substantially upregulated in SMV-infected soybean leaf tissues, suggesting that GmGSTU10-10 represents a defense-specific GST enzyme [24]. SMV is the most prevalent viral pathogen of soybean in the world. Infection by SMV usually causes yield losses of between 35 and $50 \%$ under natural field conditions and up to $50-100 \%$ in severe outbreaks [25].

Investigating plant host response at the molecular level is certainly important for control of SMV infections. GmGSTU10-10 displays an interesting expression pattern [24]. In particular, GmGSTU10-10 transcripts were significantly over-represented at 21 days post infection (p.i.). (by $>2.20$-fold), but not at 7 and 14 days p.i. This supports the idea that there is a delayed host defense response and at late infection stages, the soybean plant responds to SMV infection by expressing GmGSTU10-10. The same phenomenon has also been observed in other defense-related transcripts in the SMV-infected leaf $[24,26]$. For example, of the 24 upregulated defense-related transcripts, a subset of 17 defense-related transcripts that are involved in disease signaling, plant defense and stress responses (such as chitinase, GmGSTU10-10, heat shock protein, superoxide dismutase, peroxidase), were either downregulated or slightly affected at 7 days p.i., but substantially upregulated at 14 or 21 days p.i. [24].

The actual biological role of GmGSTU10-10 is unclear; however, additional pieces of evidence suggest that the enzyme is related to defense towards biotic stress. The biological role of GmGSTU10-10 in biotic stress is also supported by the recent finding that this enzyme is differentially expressed in soybean in response to Phakopsora pachyrhizi infections [27]. On the other hand, the enzyme is down regulated by abiotic stress (salt stress, $\mathrm{NaCl}$ ) as demonstrated using proteomic analysis [28]. It is noteworthy that GmGSTU10-10 gene was found to be constitutively expressed in soybean, suggesting that the enzyme has housekeeping roles and presumably is involved in endogenous developmental processes of soybean [29].

\section{Experimental procedures}

\subsection{Materials}

Poly(A)-mRNA purification kit, total RNA isolation kit, first-strand cDNA synthesis kits, dNTPs and restriction enzymes were obtained from Invitrogen (USA). Reduced glutathione, 1-chloro-2,4-dinitrobenzene
(CDNB), and all other enzyme substrates were obtained from SigmaAldrich (USA). The T7 expression vector pEXP5-CT/TOPO® was obtained from Invitrogen (USA).

\subsection{Methods}

\subsubsection{Molecular cloning}

Soybean seedlings were grown in perlite in a growth chamber under controlled conditions $\left(25^{\circ} \mathrm{C}, 16 \mathrm{~h}\right.$ light/ $8 \mathrm{~h}$ dark cycle). Poly(A)-mRNA was purified from plant seedlings using polyA RNA isolation kit. Purified RNA was qualitatively and quantitatively assessed by spectrophotometry and agarose gel electrophoresis. First-strand cDNA synthesis was done using oligo- $\mathrm{p}(\mathrm{dT})_{15}$ primer and AMV reverse transcriptase. In order to eliminate contamination of genomic DNA, RNA samples were treated with DNase I at $37^{\circ} \mathrm{C}$ for $45 \mathrm{~min}$. The PCR-primers 5'-ATGACA GATGAGGTGGTTCTT-3' (forward primer) and 5'-CTACTCGATGACTA ACTTCTTTCTTAT-3' (reverse primer) were designed according to the GmGSTU10-10 gene sequence [17]. The PCR reaction was carried out in a total volume of $50 \mu \mathrm{L}$ containing: 10 pmole of each primer, $50 \mathrm{ng}$ template cDNA, $0.2 \mathrm{mM}$ dNTPs, $5 \mu \mathrm{l} 10 \times$ Pfu buffer and 1 unit of Pfu extended DNA polymerase. The PCR procedure comprised 30 cycles of $1.5 \mathrm{~min}$ at $95^{\circ} \mathrm{C}, 1 \mathrm{~min}$ at $55^{\circ} \mathrm{C}$ and $2 \mathrm{~min}$ at $72{ }^{\circ} \mathrm{C}$. A final extension time at $72{ }^{\circ} \mathrm{C}$ for $10 \mathrm{~min}$ was performed after the 30th cycle. The resulting PCR amplicons were TOPO ligated into a T7 expression vector (pEXP5-CT/TOPO $\left.{ }^{\circledR}\right)$. The resulting expression constructs were sequenced along both strands and were used to transform competent Escherichia coli BL21(DE3) cells.

\subsubsection{Expression and purification of recombinant GmGSTU10-10}

E. coli cells harboring recombinant plasmid were grown at $37^{\circ} \mathrm{C}$ in $1 \mathrm{~L}$ LB medium containing ampicillin $(100 \mu \mathrm{g} / \mathrm{mL})$. The synthesis of GST was induced by the addition of $1 \mathrm{mM}$ isopropyl 1-thio- $\beta$ galactopyranoside when the absorbance at $600 \mathrm{~nm}$ reached 0.6 . Four hours after induction, cells were harvested by centrifugation at $8000 \mathrm{rpm}$ for $15 \mathrm{~min}$, resuspended in potassium phosphate buffer (0.1 M, pH 6.5), sonicated, and centrifuged at 13,000 rpm for $5 \mathrm{~min}$. The supernatant was loaded to GSH-Sepharose column (1,4-butanediol diglycidyl ether-GSH-Sepharose-CL6B, $1 \mathrm{~mL}$ ), which was previously equilibrated with potassium phosphate buffer (20 mM, pH 7). Nonadsorbed protein was washed off with $10 \mathrm{~mL}$ equilibration buffer. Bound GST was eluted with equilibration buffer containing $10 \mathrm{mM}$ GSH. Protein purity was judged by SDS-PAGE.

\subsubsection{Assay of enzyme activity and protein concentration}

Enzyme assays for the CDNB and fluorodifen conjugation reactions were performed according to published methods [16,30,31]. Observed reaction velocities were corrected for spontaneous reaction rates when necessary. Turnover numbers were calculated on the basis of one active site per subunit. Glutathione peroxidase activity was determined according to Wilson's method [32]. The reactions were carried out in $0.1 \mathrm{M}$ potassium phosphate buffer, $\mathrm{pH} 7.5$, containing $1 \mathrm{mM}$ EDTA, $1 \mathrm{mM} \mathrm{GSH}, 1.5 \mathrm{mM}$ cumene hydroperoxide $(\mathrm{CuOOH})$ or tertbutyl peroxide ( $\mathrm{t}-\mathrm{BuOOH}$ ), $0.2 \mathrm{mM}$ NADPH, 1 unit glutathione reductase, and enzyme. Protein concentration was determined by the Bradford assay using bovine serum albumin (fraction V) as standard. Michaelis-Menten equation was fitted to steady-state data by nonlinear regression analysis using the GraFit computer program (Erithacus Software Ltd.).

\subsubsection{Viscosity dependence of kinetic parameters}

The effect of viscosity on kinetic parameters was assayed based on references [30,33-35]. According to Kramers' theory [33], enzymes that undergo conformation changes during catalysis should be inhibited by viscosity [34]. In a diffusion dependent, enzyme-catalyzed reaction, where the substrate binds to the enzyme to yield the product, the $k_{\text {cat }}$ value is negatively influenced by the friction of the solvent with the 

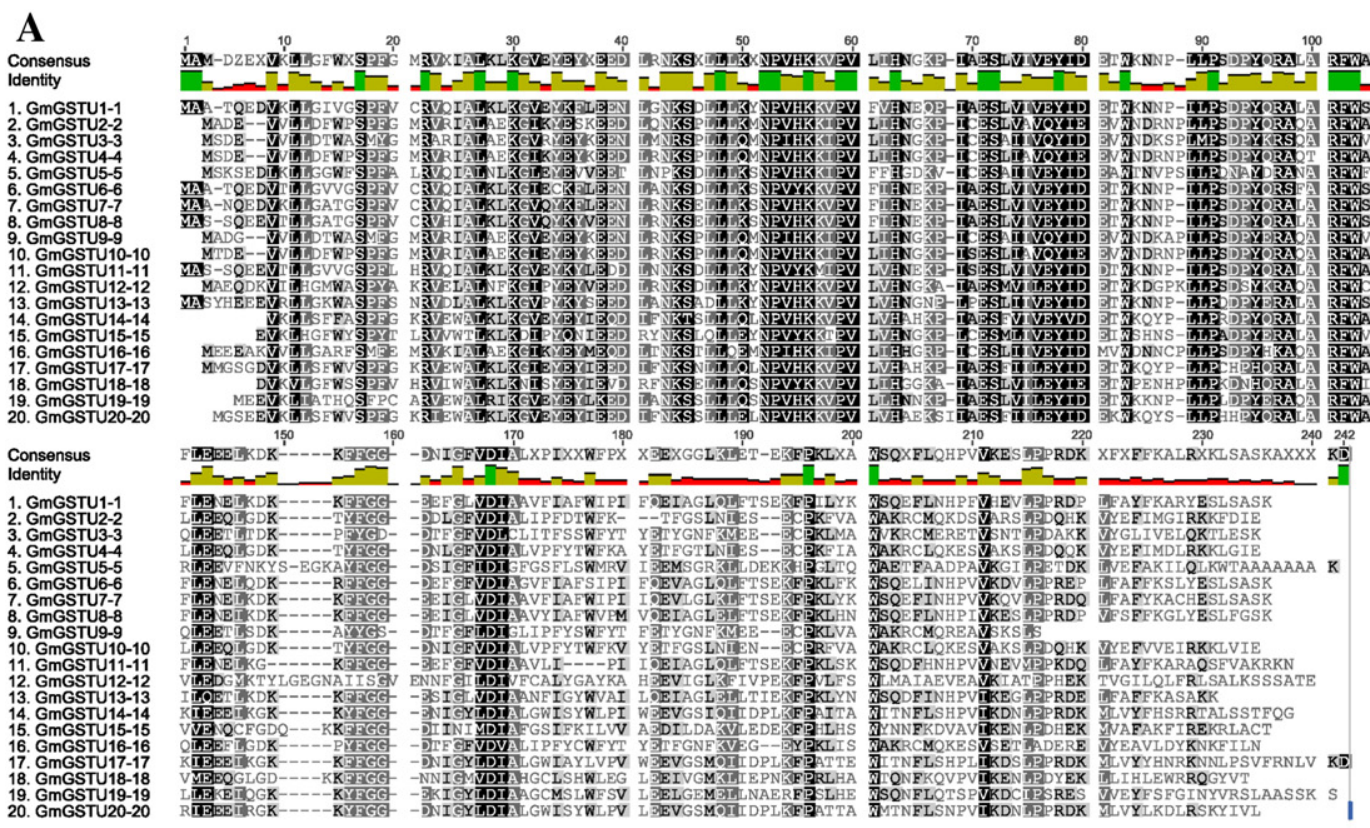

B

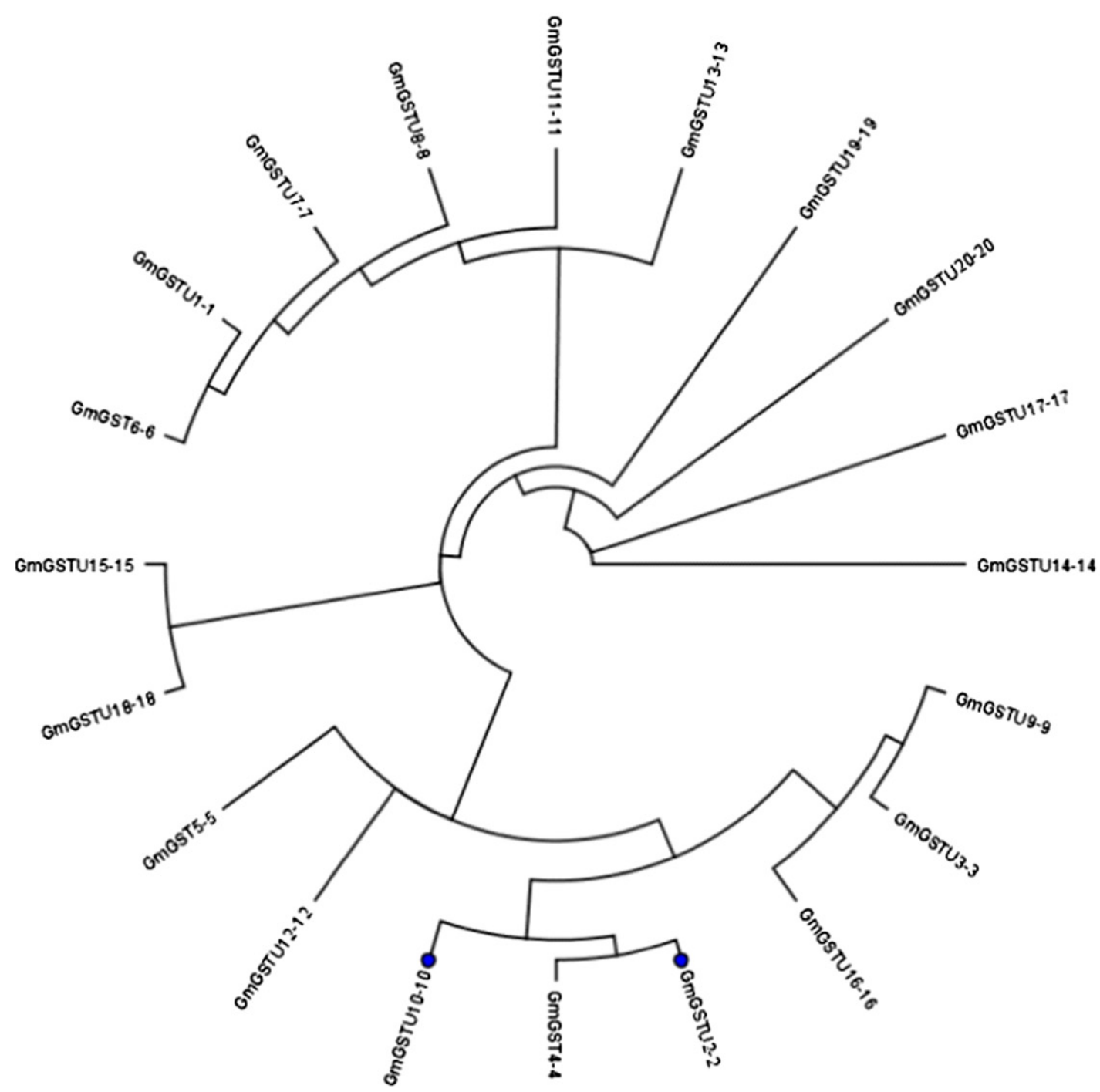


enzyme. This is because friction increases the activation energy needed to reach the transition state [35]. In turn, friction is a function of viscosity $\eta$. Thus, the reaction rate constant depends linearly on viscosity $\eta$ (Eq. (1)) as described by Jacob and Schmid [34]:

$k=\eta^{-1} e^{-\frac{\Delta U}{R T}}$

where $k$ is the rate constant for the reaction $\left(k_{c a t}\right.$ or $\mathrm{V}_{\max }$ for enzyme catalyzed reactions), $\eta$ is the macroscopic viscosity of the solvent, $R$ is the gas constant, $T$ is the absolute temperature and $\Delta U$ is the free energy barrier imposed by solvent friction.

\subsection{5. $\mathrm{pH}$ and temperature dependence of $V_{\max }$}

Analysis of the $\mathrm{pH}$ dependence of $\mathrm{V}_{\max }$ was carried out in $0.1 \mathrm{M}$ potassium phosphate buffer ( $\mathrm{pH}$ 6.0-8.5). The $\mathrm{pH}$-dependent ionizations were determined by a fit of steady-state parameters to Eq. (2),

$u=\frac{C}{1+[H] / K_{1}+K_{2} /[H]}$

where $u$ is the observed rate of the reaction, $C$ is the $\mathrm{pH}$ independent rate, $[H]$ is the concentration of hydrogen ions, and $K_{1}$ and $K_{2}$ are the ionization constants of the acid and base species, respectively [36]. pK values were calculated by the program GraFit (Erithacus Software Ltd.).

The effect of temperature on $k_{\text {cat }}$ of the CDNB conjugation reaction, the activity data were analyzed by plotting the logarithm of activity versus the reciprocal of the absolute temperature. Arrhenius equation was fitted to the experimental data (Eq. (3)) [37,38]:

$\log k_{c a t}=\log Z-\frac{E_{a}}{2.303 R T}$

where $E_{a}$ is the energy of activation, $R$ is the gas constant, and $Z$ is the preexponential factor. The Eyring equation was fitted to the activity data (Eq. (4)) [39]:

$k_{\text {cat }}=\frac{k_{\beta} T}{h} e^{-\left(\frac{\Delta G^{\neq}}{R T}\right)}=\frac{k_{\beta} T}{h} e^{-\left(\frac{\Delta H^{\neq}}{R T}-\frac{\Delta S^{\neq}}{R}\right)}$

where $k_{\beta}$ is the Boltzmann's constant, $h$ is Planck's constant, $R$ is the gas constant, and $\Delta \mathrm{G}^{\neq}, \Delta \mathrm{H}^{\neq}$and $\Delta \mathrm{S}^{\neq}$are the free energy, enthalpy, and entropy of activation of the rate limiting step in the reaction, respectively. The data were plotted as logarithm of $k_{\text {cat }} / T$ versus the reciprocal of the absolute temperature.

\subsubsection{Thermal stability}

GmGSTU10-10 was incubated at different temperatures at a protein concentration of $0.02 \mathrm{mg} \mathrm{mL}^{-1}$ in $0.1 \mathrm{M}$ potassium phosphate buffer $\mathrm{pH}$ 7. The samples were incubated at different temperatures $\left(20\right.$ to $85^{\circ} \mathrm{C}$ ) for $5 \mathrm{~min}$ and subsequently assayed for residual activity. $\mathrm{T}_{\mathrm{m}}$ values were determined from the plot of relative inactivation (\%) versus temperature $\left({ }^{\circ} \mathrm{C}\right)$. The $\mathrm{T}_{\mathrm{m}}$ value is the temperature at which $50 \%$ of the initial enzyme activity is lost after heat treatment.

\subsubsection{Crystallization and data collection and processing}

GmGSTU10-10 was concentrated to $10 \mathrm{mg} / \mathrm{mL}$ in buffer HEPES$\mathrm{NaOH}$ pH 7.0 prior to crystallization. Initial crystallization conditions were established using the INDEX crystallization screen (Hampton

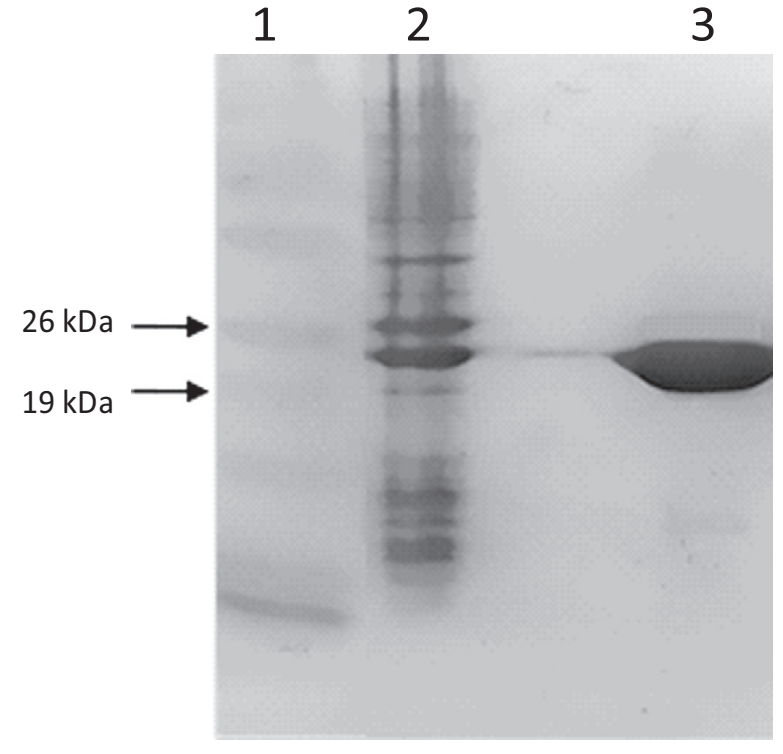

Fig. 2. SDS-PAGE analysis of purified GmGSTU10-10 enzyme. Lane 1: Molecular weight markers. Lane 2: Crude extract of $E$. coli BL21(DE3) expressing GmGSTU10-10. Lanes 3: Eluted fraction from the affinity column.

Table 1

Steady-state kinetic analysis of GmGSTU10-10.

\begin{tabular}{lccl}
\hline Substrate & $\begin{array}{l}k_{\text {cat }} \\
\left(\mathrm{min}^{-1}\right)\end{array}$ & $\mathrm{K}_{\mathrm{m}}(\mu \mathrm{M})$ & $\begin{array}{l}k_{\text {cat }} / \mathrm{K}_{\mathrm{m}} \\
\left(\mu \mathrm{M}^{-1} \mathrm{~min}^{-1}\right)\end{array}$ \\
\hline GSH & $159 \pm 12.2$ & $67.9 \pm 4.5$ & 2.35 \\
CDNB & & $280 \pm 22.2$ & 0.57 \\
GSH & $35 \pm 2.1$ & $28.6 \pm 1.6$ & 1.22 \\
CuOOH & & $252 \pm 20.2$ & 0.14 \\
GSH & $15.8 \pm 0.8$ & $87.0 \pm 5.5$ & 0.18 \\
t-BuOOH & & $853 \pm 45.5$ & 0.02 \\
GSH & $49.1 \pm 2.2$ & $64.8 \pm 4.3$ & 0.76 \\
Fluorodifen & & $66.2 \pm 4.4$ & 0.74 \\
\hline
\end{tabular}

Research) in the presence of $10 \mathrm{mM}$ spirodiclofen dissolved in acetone solution. Condition No. $82\left(0.2 \mathrm{M} \mathrm{MgCl}_{2}, 0.1 \mathrm{M}\right.$ Bis-Tris, pH 5.5, PEG $335025 \%(w / v))$ resulted in the appearance of small crystals. After optimization, crystals of maximum size of $0.4 \mathrm{~mm} \times 0.2 \mathrm{~mm} \times 0.2 \mathrm{~mm}$ were grown within 2 days in $0.2 \mathrm{M} \mathrm{MgCl}_{2}, 0.1 \mathrm{M}$ Bis-Tris, pH 5.6, PEG 3350 $22 \%(w / v)$ using the hanging drop vapor diffusion method. X-ray diffraction data to $1.6 \AA$ resolution were collected from a single crystal at $100 \mathrm{~K}$ on station X13 ( $\lambda=0.8123 \AA$ ) at EMBL Hamburg, c/o DESY, Germany, using a $165 \mathrm{~mm}$ MARCCD detector. All data were autoindexed, integrated, and scaled using the XDS software package [40].

\subsubsection{Structure determination and refinement}

The structure was determined by molecular replacement using PHASER [41] in the CCP4 program suite [42]. The subunit A of GST from G. max in complex with GSH (PDB ID: 3FHS, 91\% sequence identity with GmGSTU10-10 [43]) was used as a search model after the waters,

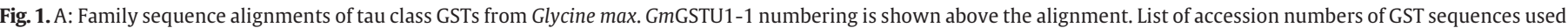

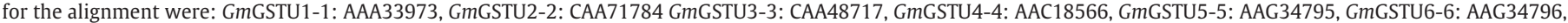

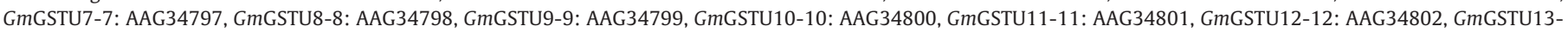

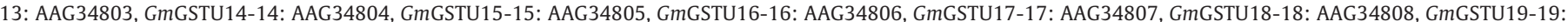

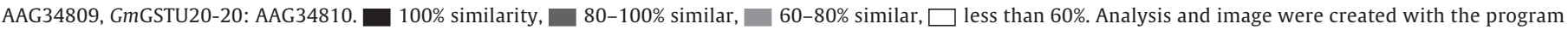

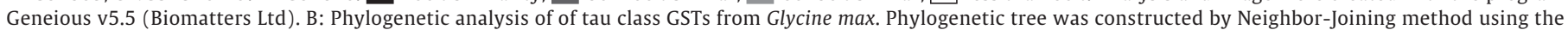
Geneious v5.5 program (Biomatters Ltd). The tree was formed after alignment of the protein sequences using ClustalW [61]. 
side chains and the ligand were removed. The resulting top solution from PHASER showed a Z-score of 50.7. After calculation of an initial electron-density map, most of the side chains were visible in the model and manual building was carried out. The program COOT [44] was used for inspection of $2\left|\mathrm{~F}_{\mathrm{o}}\right|-\left|\mathrm{F}_{\mathrm{c}}\right|$ and $\left|\mathrm{F}_{\mathrm{o}}\right|-\left|\mathrm{F}_{\mathrm{c}}\right|$ maps, and rebuilding of the structure. Refinement was carried out with PHENIX [45]. A randomly selected subset (5\%) of the total number of reflections was set aside for cross-validation analysis to monitor the progress of refinement using the $\mathrm{R}_{\text {free }}$ factor. The quality of the final structure was assessed with MOLPROBITY [46], PROCHECK [47], and validation tools in COOT.

\subsubsection{Protein Data Bank accession code}

The refined coordinates of GmGSTU10-10 and the structure factors have been deposited with the Protein Data Bank (PDB ID: 4CHS).
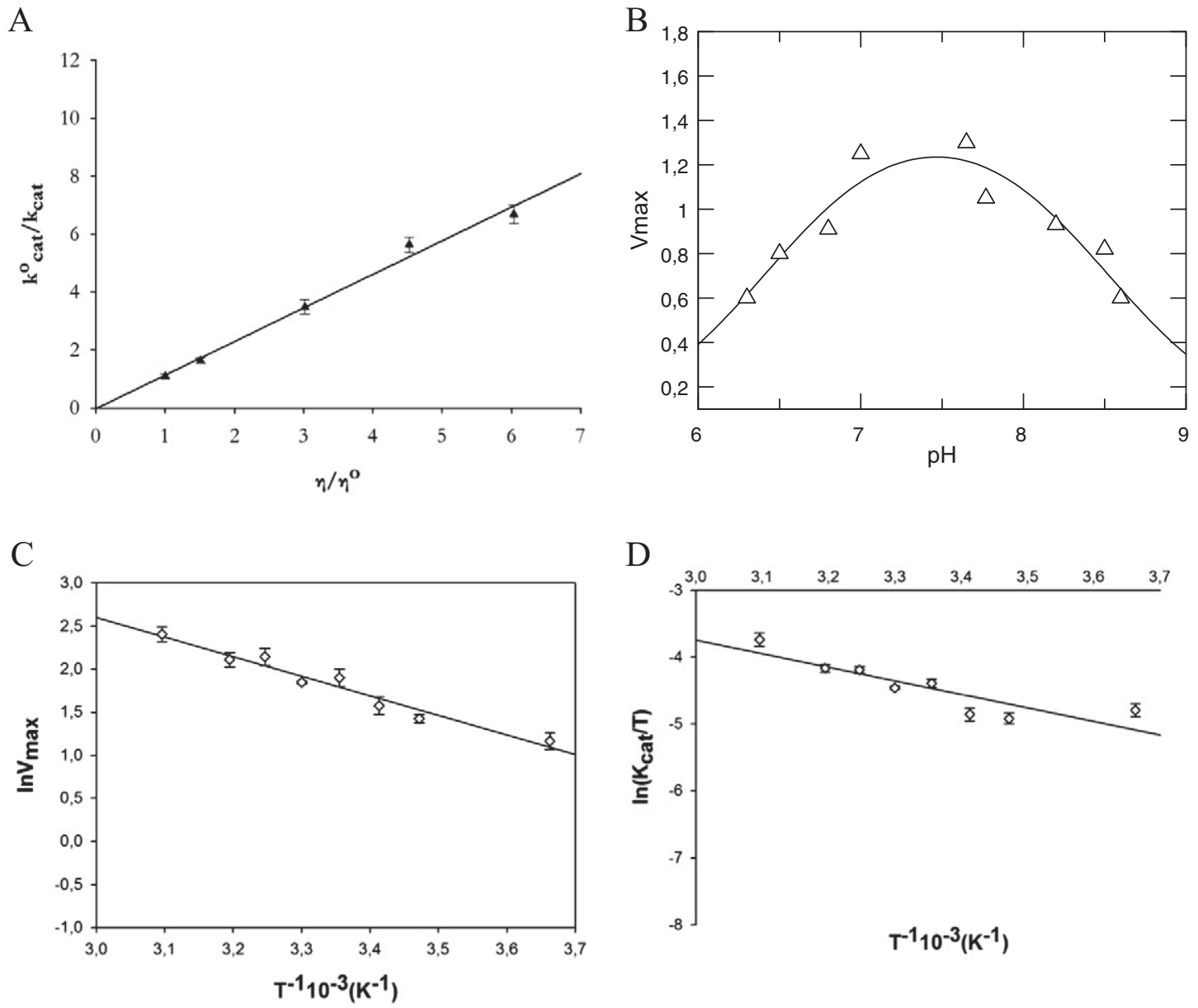

$\mathrm{D}$
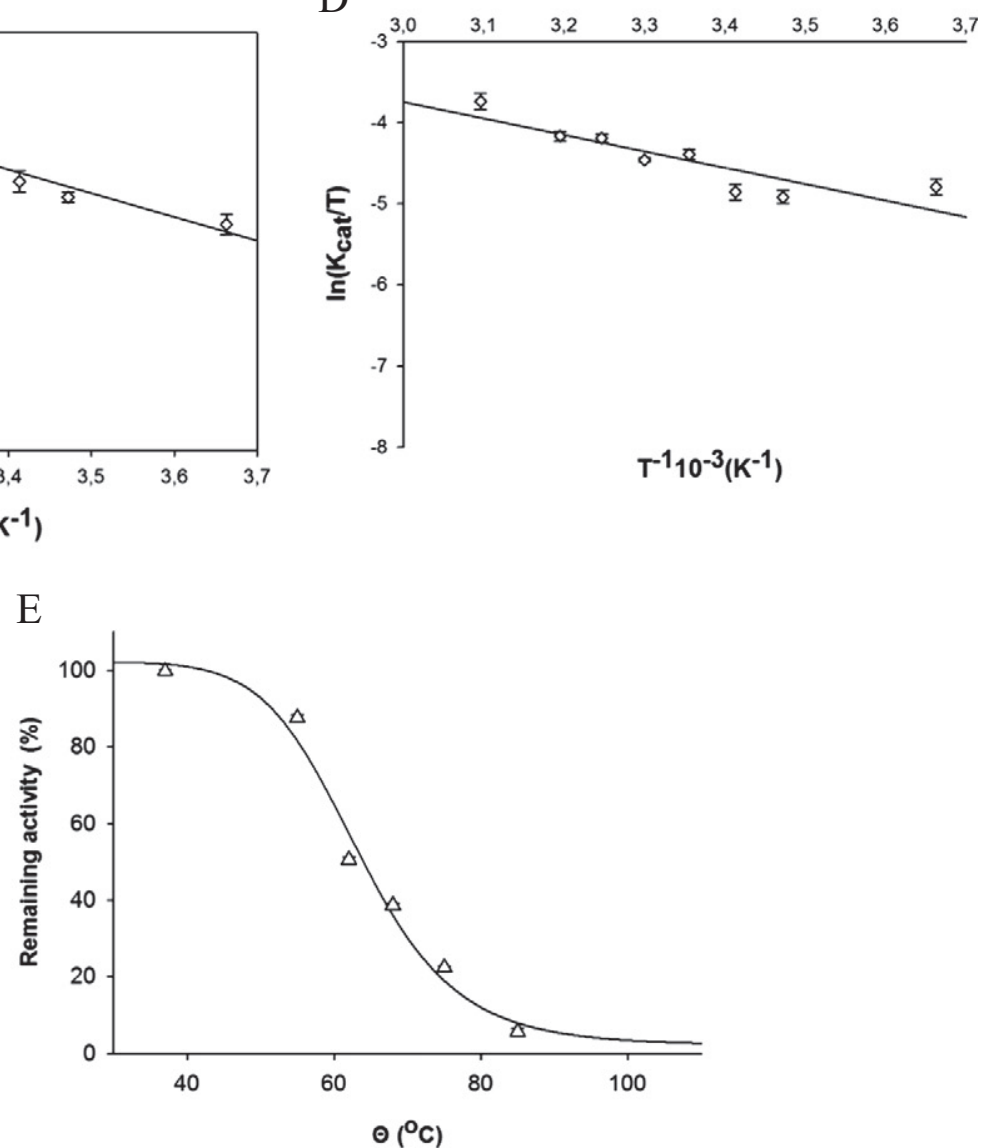

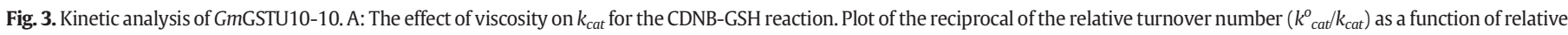

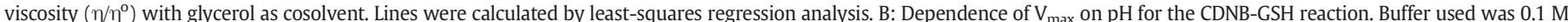

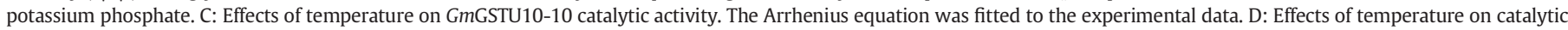

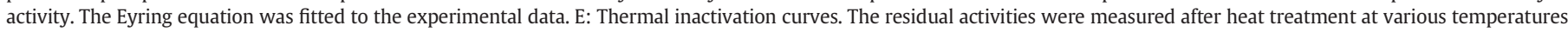
$\left({ }^{\circ} \mathrm{C}\right)$ for $5 \mathrm{~min}$. 


\subsubsection{Bioinformatics and structural analysis}

The superimposed structures were visually inspected using COOT. Contacts were measured with the program CONTACT in CCP4 [48]. The interface analysis was done with PDBePISA server (http://www. ebi.ac.uk/msd-srv/prot_int/pistart.html) [49]. The secondary structure assignments were produced with DSSP [50]. Calculation of enzyme area and volume occupied by each active site was carried out using CASTp [51].

\section{Results and discussion}

\subsection{Cloning, expression and kinetic characterization of the recombinant GmGSTU10-10 enzyme}

The coding sequence of GmGSTU10-10 with complete open reading frame was isolated using RT-PCR. GmGSTU10-10 contained an open reading frame (ORF) of $660 \mathrm{bp}$, coding for a polypeptide of 219 amino acid residues with a predicted molecular mass of 25,578.55 Da and a theoretical pI of 5.71. In silico analysis, using iPSORT, TargetP and SignalP algorithms, revealed the absence of putative $\mathrm{N}$-terminal transit peptide, suggesting that it is a cytosolic enzyme. The deduced amino acid sequence shares high homology with the tau class plant GSTs. Fig. 1 depicts the phylogenetic relationship of GmGSTU10-10 with other tau class GSTs from G. max. The GmGSTU10-10 is phylogenetically the closest with the isoenzymes GmGSTU2-2 and GmGSTU4-4 and shares > $88 \%$ sequence identity.

The coding sequence of GmGSTU10-10 was TOPO ligated into a T7 expression vector. The resulting expression construct was used to transform competent E. coli BL21(DE3) cells. The recombinant enzyme was purified to homogeneity by affinity chromatography on GSH-Sepharose column (Fig. 2). The substrate specificity of the purified GmGSTU10-10 was investigated using steady-state kinetic analysis. The enzyme was assayed for activities as transferase and peroxidase, using four selected substrates (CDNB, fluorodifen, cumene hydroperoxide and tert-butyl hydroperoxide). The catalytic activity of the enzyme varies widely with different substrates (Table 1). This may be used as a rationale to suggest that multiple GSTs allow for flexibility in detoxifying a wide range of potential

Table 2

Data collection and refinement statistics.

\begin{tabular}{|c|c|}
\hline \multicolumn{2}{|l|}{ Data collection } \\
\hline Space group & $P 2_{1} 2_{1} 2_{1}$ \\
\hline Cell dimensions $(\AA)$ & $\mathrm{a}=47.7 \mathrm{~b}=90.9 \mathrm{c}=112.9$ \\
\hline No of molecules & 2 \\
\hline Resolution range $(\AA)$ & $20.0-1.6$ \\
\hline Number of measured reflections & 226,980 \\
\hline Unique reflections & 65,115 \\
\hline Completeness (\%) & $99.5(98.9)$ \\
\hline Mosaicity $\left({ }^{\circ}\right)$ & 0.5 \\
\hline$<\mathrm{I} / \sigma(\mathrm{I})>$ & $17.8(2.6)$ \\
\hline$R_{\text {merge }}, R_{\text {meas }}$ & $0.045(0.455), 0.053(0.543)$ \\
\hline Wilson B-factor $\left(\AA^{2}\right)$ & 26.7 \\
\hline \multicolumn{2}{|l|}{ Refinement } \\
\hline Reflections (working/test) & $61,859 / 3256$ \\
\hline$R_{\text {cryst }}(\%), R_{\text {free }}(\%)$ & $16.2,19.4$ \\
\hline No of protein atoms & 3561 \\
\hline No of waters & 764 \\
\hline No of ligand atoms & 42 \\
\hline Bond lengths $(\AA)$ & 0.012 \\
\hline Bond angles $\left({ }^{\circ}\right)$ & 1.39 \\
\hline Residues in most favorable regions (\%) & 97.4 \\
\hline Residues in additional allowed regions (\%) & 2.1 \\
\hline \multicolumn{2}{|l|}{ Average $B$ factors $\left(\AA^{2}\right)$} \\
\hline Protein & 19.7 \\
\hline Waters & 34.2 \\
\hline GSOH & 16.9 \\
\hline
\end{tabular}

Numbers in parentheses correspond to the highest resolution shell (1.7-1.6 $\mathrm{\text { }}$ ). allochemicals and xenobiotics. The $\mathrm{K}_{\mathrm{m}}$ values for the xenobiotic substrates range between 66.2 and $853.3 \mu \mathrm{M}$, and the catalytic efficiency $\left(k_{\text {cat }} / \mathrm{K}_{\mathrm{m}}\right)$ differs by 37 -fold. GmGSTU10-10 exhibits high hydroperoxidase activity. Among all peroxides assayed, cumene hydroperoxide was the best substrate for GmGSTU10-10. The high catalytic efficiency of GmGSTU10-10 towards organic hydroperoxides may provide a link to the in vivo functional role of the enzyme towards oxidative-stress tolerance. Reactive or activated oxygen species have been suggested to be key mediators of local and systemic resistance responses in incompatible plant-pathogen reactions and to be involved in symptom development and pathogenesis in compatible plant-virus interactions [52]. The expression of GmGSTU10-10 [24] is suppressed significantly in the SMV-infected leaf at 14 days p.i. and significantly over-represented at 21 days p.i. (by $>2.20-$ fold). On the other hand, the enzyme peroxidase is induced significantly 14 days p.i. (by $>4$-fold) and downregulated ( $>-9$-fold) at 21 days p.i. This mechanism of upregulation of peroxidise transcripts and downregulation of GST transcripts may provide adjustment to the oxidative stress in the early or late infection process. Such an antioxidative metabolism imbalance may be associated with the progression of SMV infection and symptom development, as suggested for the plum pox virus-peach interaction [52].

The $K_{m}$ values for GSH, using different electrophilic substrates, lie between a relative narrow range $(28.6-87.01 \mu \mathrm{M})$. The $\mathrm{K}_{\mathrm{m}}$ values for GSH are about 5-10-times lower compared to other tau class GST isoenzymes, as for example the isoenzyme GmGSTU4-4 [16] or the Phi class enzyme ZmGSTF1-1 [30,53], but is close to that observed for the biotic stress inducible glutathione transferase from Phaseolus vulgaris [11]. The low $\mathrm{K}_{\mathrm{m}}$ suggests that GmGSTU10-10 is able to work efficiently under oxidative stress conditions where the concentration of reduced GSH is low [54]. It therefore appears that evolutionary selection pressures have optimized the kinetic parameter of the GmGSTU10-10 towards conditions under which the enzyme is induced for exploring its biological role.

The effect of viscosity on the kinetic parameters of GmGSTU10-10 was measured in order to analyze the rate-limiting step of their catalytic reaction (Fig. 3A). It is well accepted that during catalysis in GSTs, conformational changes occur, at least in the active site [21]. Furthermore, many GSTs during catalysis exhibit widely different structural conformations and induced fit mechanism $[21,43,55,56]$. At a fixed temperature, any increase in viscosity would be expected to result in an increase in free energy $[34,35]$. To assess whether the catalytic activity of the GmGSTU10-10 is influenced by the viscosity, the activity data $k_{\text {cat }}^{o} / k_{\text {cat }}$ were plotted against the solvent relative viscosity, defined as $\eta / \eta^{o}$ (see Eq. (1)). In these calculations, $k_{\text {cat }}^{o}$ and $\eta^{o}$ are respectively the $k_{\text {cat }}$ and the viscosity in the absence of glycerol and $k_{\text {cat }}$ and $\eta$ are the observed values at each glycerol concentration. A plot of the inverse relative rate constant $k_{\text {cat }}^{o} / k_{\text {cat }}$ versus the relative viscosity $\eta / \eta^{o}$ should be linear, with a slope equals to unity when the product release is limited by a strict diffusional barrier or close to zero if the catalytic reaction chemistry is rate-limiting $[16,53]$. The inverse relative rate constant $k_{\text {cat }}^{o} / k_{\text {cat }}$ for GmGSTU10-10 shows linear dependence on the relative viscosity with a slope $1.106 \pm 0.007$ (Fig. 3A). The slope is close to unity, suggesting that the product release is limited by a strict diffusional barrier. In the case of the homologue enzyme GmGSTU4-4, the dependence on the relative viscosity showed a slope $0.87 \pm 0.1$ suggesting that the rate-limiting step in the GmGSTU4-4 is not dependent on a diffusional barrier and other viscosity-dependent motions or conformational changes of the protein contribute to the rate-limiting step of the catalytic reaction [3].

The $\mathrm{pH}$ dependence of $\mathrm{V}_{\max }$ for GmGSTU10-10 was investigated in an attempt to compare the acid/base properties of the ionizable groups upon substrate binding and catalysis. The $\mathrm{pH}$ dependence of $\mathrm{V}_{\max }$ was determined over the $\mathrm{pH}$ range of 6.0 to 8.5 . The $\mathrm{pH}-\mathrm{V}_{\max }$ profiles are shown in (Fig. 3B). The activity of GmGSTU10-10 was greatest between pH 7 and 8, indicating a broad pH optimum. Eq. (2) was fitted to the 

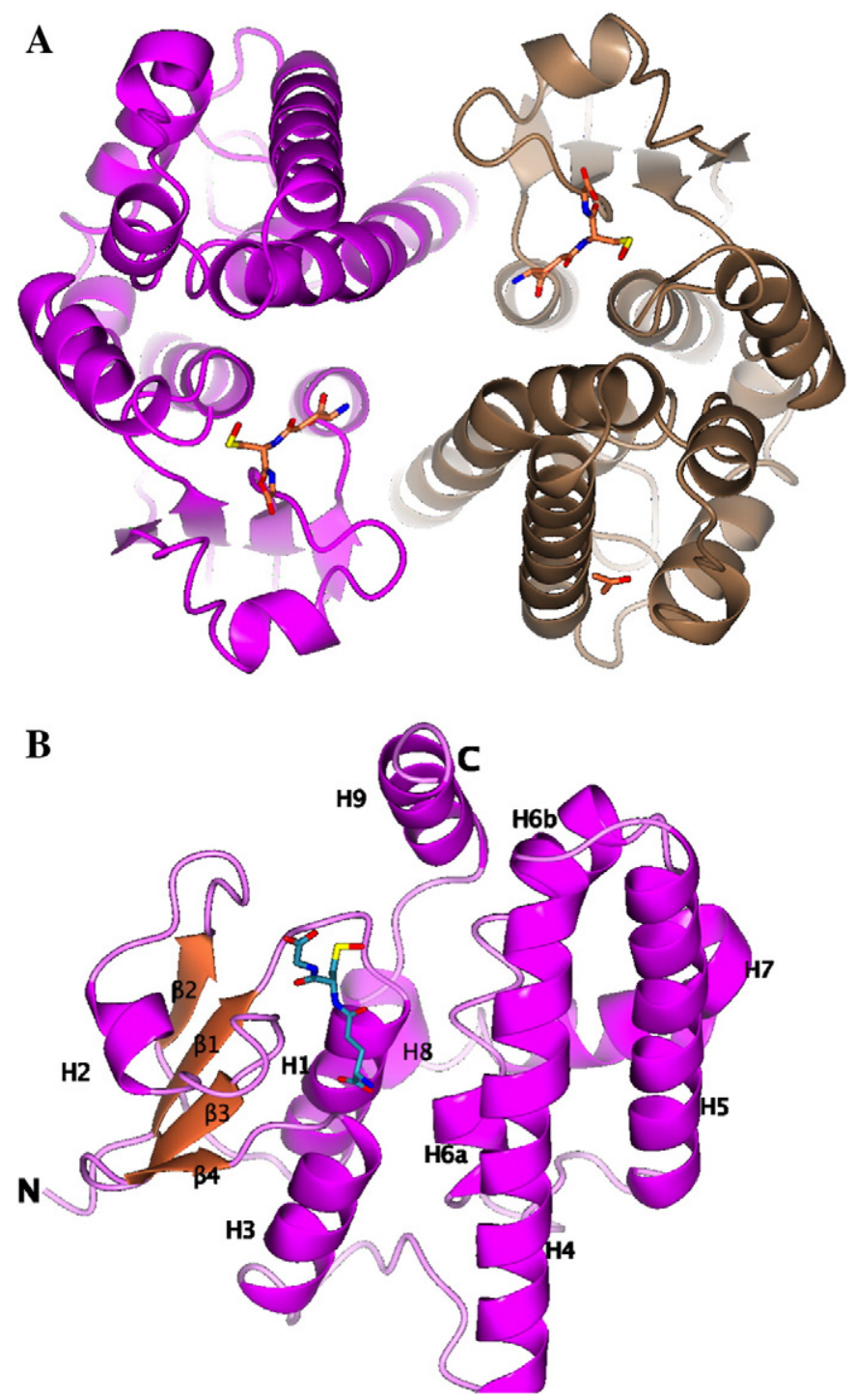

Fig. 4. A: Ribbon diagram of the GmGSTU10-10 dimer. Subunit A and B are colored magenta and brown, respectively. GSOH and acetone are represented as sticks and are colored according to atom type. B: Ribbon diagram of the GmGSTU10-10 monomer. GSOH bound at the active site is represented as stick and is colored according to atom type. The helices are colored magenta, strands in coral and turns in pink.

data, giving $\mathrm{pK}_{1}=6.5 \pm 0.2$ and $\mathrm{pK}_{2}=8.4 \pm 0.2$. The $\mathrm{pK}_{1}$ is close to the value expected for a carboxylate side chain, or for the imidazole group of a His residue [57], whereas, the $\mathrm{pK}_{2}$ is close to the value expected for Lys or Cys residues [57]. The crucial property of GSTs is their ability to lower the $\mathrm{pK}_{\mathrm{a}}$ of the thiol group of the bound GSH. In the tau class GSTs this is accomplished by the active site Ser whose hydroxyl group of the sidechain forms a hydrogen with the $-\mathrm{SH}$ group. Published work shows that the pKa of GSH in the active site ranges from 6.0 to 6.5 [30]. Presumably, the observed $\mathrm{pK}_{1}$ may reflect the ionization of the thiol group of the bound GSH.

To study the effect of temperature on $k_{\text {cat }}$, the Arrhenius and Eyring equations (Fig. 3C and D) were fitted to the experimental data and typical linear relationships were obtained indicating that the same kinetic step remains rate limiting throughout the different temperatures. The activation energy, a measure of the energy barrier that the reactants must overcome before products can be formed, was estimated equals to $19.1 \pm 1.4 \mathrm{~kJ} / \mathrm{mol}$, close to that observed for other GSTs [58]. The enzyme exhibits large negative $\Delta S^{\neq}$value $(-0.17 \pm 0.01 \mathrm{~kJ} / \mathrm{mol} . \mathrm{K})$, which is characteristic of transition states that are more ordered than the corresponding ground states with minimal disruption of the water network in the active center of the enzymes. As expected, a positive $\Delta \mathrm{H}^{\neq}$ was observed $(12.4 \pm 1.1 \mathrm{~kJ} / \mathrm{mol})$ because the transition state involves the breaking of bonds. The fairly positive value of $\Delta \mathrm{H}^{\neq}$indicates that the transition state is highly solvated.

The thermal stability of the GmGSTU10-10 enzyme was assessed by measuring their residual activity after heat treatment for 5 min at various temperatures $\left(20-85{ }^{\circ} \mathrm{C}\right.$, Fig. $3 \mathrm{E}$ ). The $\mathrm{T}_{\mathrm{m}}$ was measured equal to $62.43 \pm 0.62{ }^{\circ} \mathrm{C}$. This $\mathrm{T}_{\mathrm{m}}$ value is similar to that determined for other tau class GST isoenzymes [3]. The absence of substantial differences in $\mathrm{T}_{\mathrm{m}}$ values suggests that the isoenzymes display similar structural stability and suggest that the potential of a GST gene to evolve new catalytic function upon evolution may depend on its ability to accept mutations without losing the stability of the protein domain that is encoded.

\subsubsection{Structure determination of GmGSTU10-10 and quality of the final model}

The structure of GmGSTU10-10 was determined to 1.6 Å resolution in complex with GSOH. The final statistics for data collection and structure refinement are summarized in Table 2. Although crystals were produced in the presence of spirodiclofen, no binding of spirodiclofen was found after inspection of the electron density maps. Instead, a GSH molecule was initially placed at the active site based on the electron density. At the later stages of refinement, GSH was replaced by GSOH owing to extra electron density near the sulfur atom. At the N-terminus, the first residue (Met1) from subunit A, the first three residues (Met1, Thr2 and Asp3) from subunit B, the last 3 residues at the C-terminus (Val217, Ile218 and Glu219) from subunit A and the last residue (Glu219) from subunit B could not be modeled in the structure owing to lack of sufficient density.

\subsubsection{Overall structure}

The structure of GmGSTU10-10 is a homodimer with a 2-fold symmetry between the subunits (Fig. 4). The dimer has a globular shape with a solvent-accessible deep V-shaped crevice at the inter-subunit interface. Hydrogen bonds, salt bridges and hydrophobic interactions are observed in the subunit-subunit interface. Structural comparison of the subunits showed only minor differences (RMSD $=0.63 \AA$ ) with respect to their secondary structure elements. Each subunit folds into two distinct domains, namely, the N-terminal domain [residues 1-77] and the all-helical C-terminal domain [residues 89-219]. The Nterminal domain adopts a thioredoxin-like fold (Fig. 4B) as in previously reported structures $[16,43]$. Helix $\mathrm{H} 2$ and strand $\beta 3$ are connected by a loop containing a cis-Pro, which is highly conserved in all GSTs (Fig. 1A). The cis-Pro loop, although not directly involved in catalysis, is important in maintaining the protein in a catalytically competent conformation [59]. The C-terminal domain consists entirely of $\alpha$ helices positioned downstream the thioredoxin structure and is connected to the $\mathrm{N}$-terminal domain by a short linker sequence.

Subunit A lacks the C-terminal residues and subunit B lacks the $\mathrm{N}$ terminal residues due to their flexible character that results in weak or no electron density at all. GSOH was found bound in both subunits. An acetone molecule was located on the surface of subunit B, in a pocket formed by Glu178, Lys126, and Ile129. This compound was used during co-crystallization in the presence of the pesticide spirodiclofen.

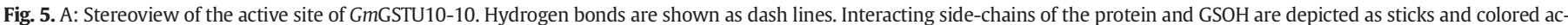

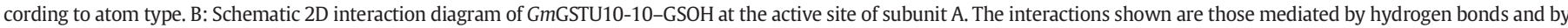

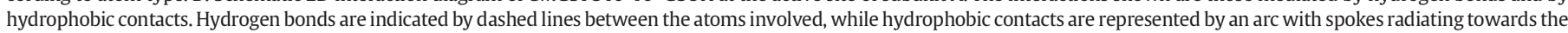
ligand atoms they contact. The contacted atoms are shown with spokes radiating back. The figure was generated using LigPlot + [62]. GS8 is the code used by PDB for GSOH. 

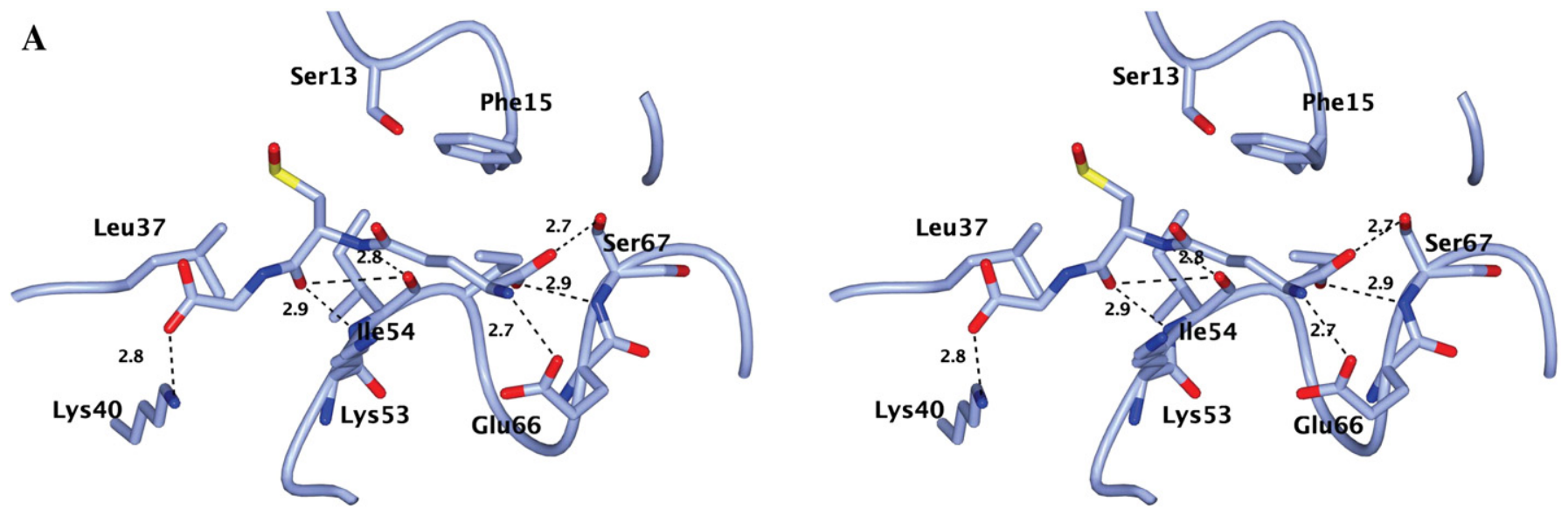

B

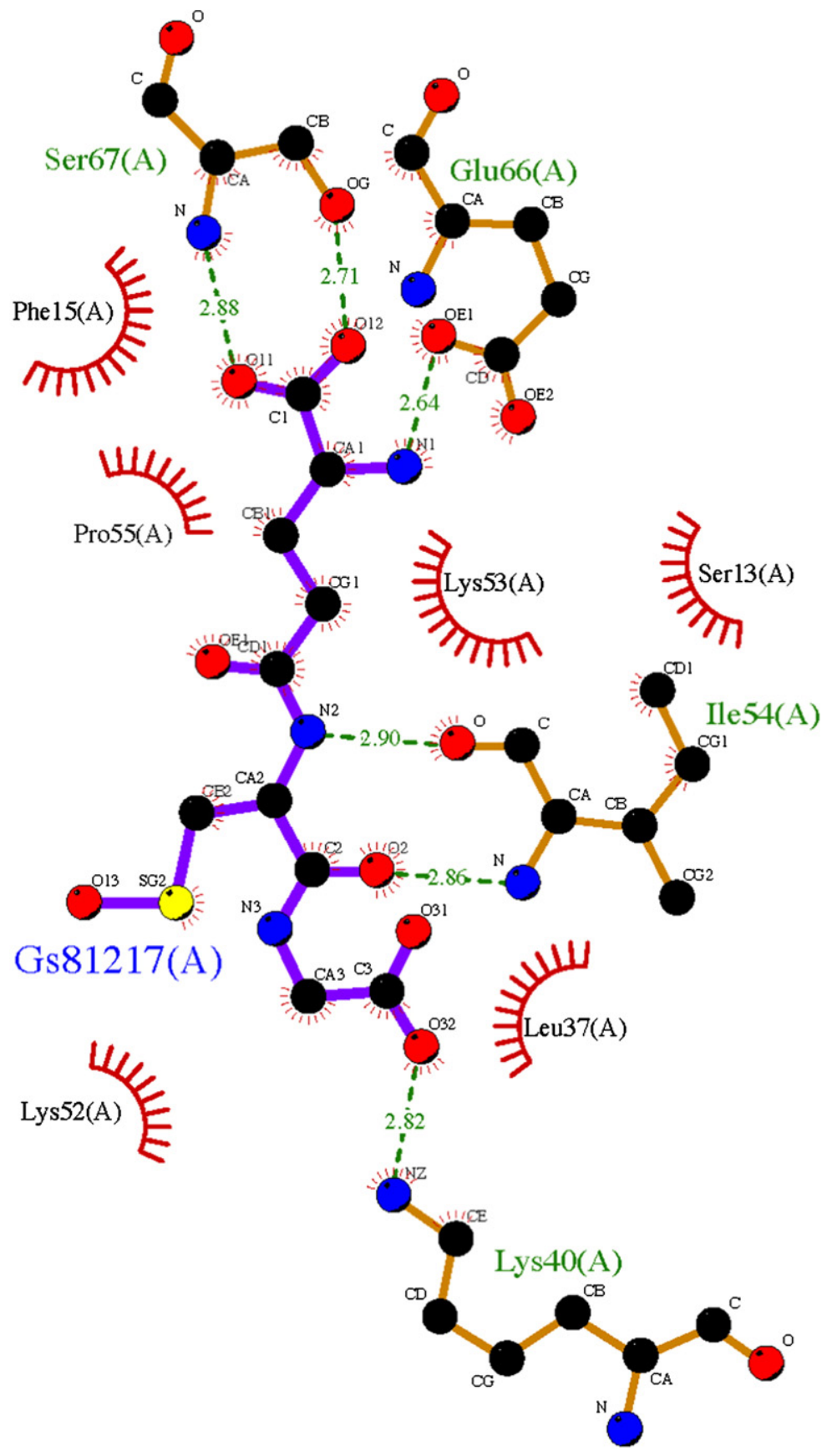



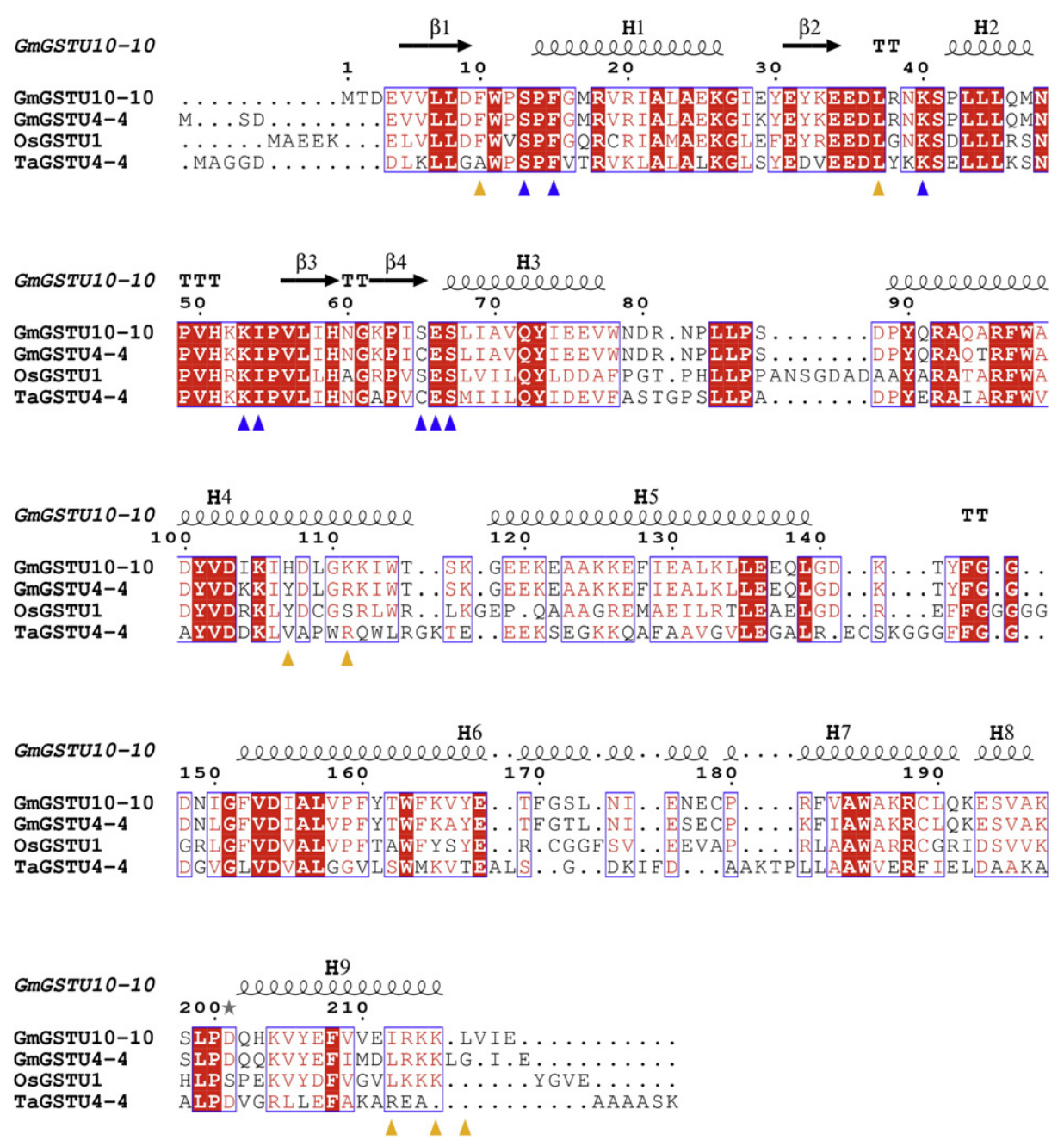

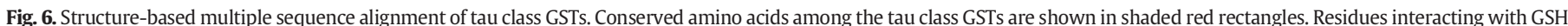

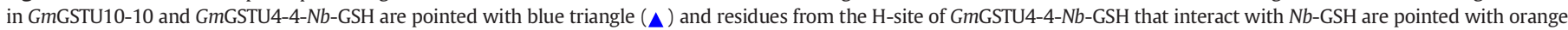

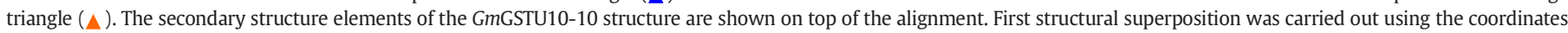

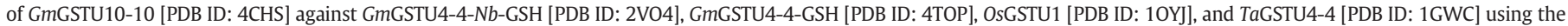
PDBeFold (http://www.ebi.ac.uk/msd-srv/ssm) server. The figure was generated using ESPript [63].

\subsubsection{GSH binding site ( $G$-site)}

In each subunit, one molecule of glutathione sulfenic acid (GSOH) was placed (Fig. 5). The GSOH was presumably formed during crystallization after oxidation of the - SH group of GSH. Naturally, GSOH is formed as an intermediate by the reaction of GSH with hydroperoxides [60]. In particular, the reaction of GSH with hydroperoxides is achieved in two steps. The first step involves the nucleophilic attack by $\mathrm{GS}^{-}$on hydroperoxide that results in the formation of GSOH. This sulfenic acid then reacts non-enzymically with GSH to produce glutathione disulfide (GSSG). Therefore, GSOH may be considered as a possible intermediate in the catalytic reaction with hydroperoxides.

As shown in Fig. 5, GSOH binds in the same conformation in both subunits. The glycine moiety of GSH is located in a polar region, formed by the beginning of helices H1 [Pro14-Lys26], H2 [Pro42-Met47], and $\mathrm{H} 3$ [Ser67-Val77] in the N-terminal domain. There is no direct interaction between the GSH portion and residues of the $\mathrm{C}$-terminal domain. Its $\gamma$-Glu moiety points downwards to the internal cavity and its glycine moiety is oriented upwards and projects into the bulk solvent. The $\gamma$ Glu moiety of GSOH forms hydrogen bonds with Glu66 and the hydroxyl group of Ser67. The complete conservation of this Ser residue (Fig. 1A) is consistent with its critical role in GSH binding [58]. The cysteinyl moiety of GSOH forms a hydrogen bond with the peptide bond of Ile54. The glycyl moiety of GSOH forms a hydrogen bond with the side chain of Lys40. In addition, the hydroxyl group of Ser13 is 4.6 $\AA$ A away from the sulfur group of the GSOH, indicating that Ser13 corresponds to the catalytic residue as in other tau class GSTs [3]. Ser13 is highly conserved and it is well established that it plays a crucial role in the mechanism of GSH activation as a catalytically essential residue [3, $10,16]$. In particular, the Ser hydroxyl group acts as hydrogen bond donor to the thiol group of GSH, contributing to stabilization of the reactive thiolate anion, which is the nucleophile group for the electrophilic substrate $[16,43]$.

\subsubsection{Electrophilic binding site ( $\mathrm{H}$-site)}

The H-site of GmGSTU10-10 resides next to the G-site and is formed by residues from the $\mathrm{C}$-terminal domain. In general, the H-site of GSTs displays a low degree of sequence identity. This reflects differences in substrate specificity, compared to other tau class plant enzymes $[3,10$, 11,16], and suggests that the C-terminal domain of these enzymes may have evolved under differential selective pressures. The H-site of GmGSTU10-10 is typically hydrophobic and built mainly by residues from the C-terminal domain: H4 (His107, Lys111, Trp114, Thr115), H6 (Trp163), H9 (Phe208 and Leu212), and Phe10 from the N-terminal domain. All these residues are oriented towards the center of the active site. These residues are not conserved among other GSTs and may play a role in regulation of substrate recognition by influencing the 

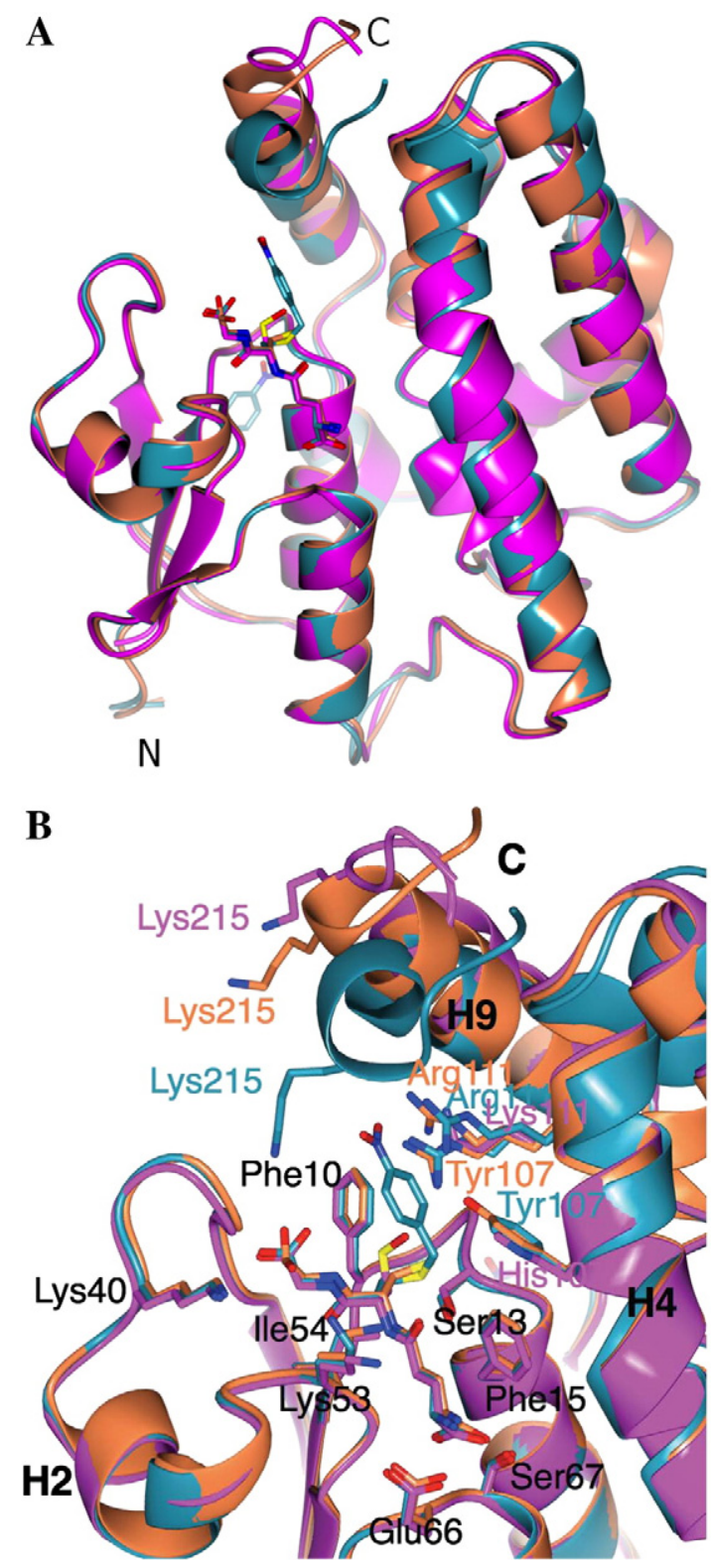

Fig. 7. A: Structural superposition of GmGSTU10-10 (magenta), GmGSTU4-4 (coral), and GmGSTU4-4-Nb-GSH (dark cyan). GSH, GSOH and Nb-GSH are shown as sticks and colored according to atom type. B: Close-up view of the side chains of GmGSTU10-10 (magenta), GmGSTU4-4 (coral), and GmGSTU4-4-Nb-GSH (dark cyan) at the active site. GSH, GSOH and $\mathrm{Nb}$-GSH are shown as sticks and colored according to atom type. Labels for Tyr107, His107, Arg111, Lys111 and Lys215 are colored according to the respective structure.

binding mechanisms and physiochemical properties of the H-site. An interesting characteristic of this site is the position of His107, which is a non-conserved residue, replaced by either polar or non-polar residues in other tau class GSTs. The side-chain of His107 is pointing towards the active site and is approximately $5.5 \AA$ away from GSOH.

\subsection{Structure comparison of GmGSTU10-10 and GmGSTU4-4}

Recently, we reported the structural and kinetic properties of the isoenzyme GmGSTU4-4 from G. $\max [3,16,43]$. This isoenzyme together with GmGSTU10-10 forms a subgroup of highly homologues enzymes (>88\% sequence identity) (Fig. 1 ) with distinct structural and functional features. Comparative structural analysis of GmGSTU10-10 and GmGSTU4-4 (in complex with GSH (GmGSTU4-4-GSH) [43] and S-pnitrobenzyl-GSH (GmGSTU4-4-Nb-GSH) [16]) was used to identify key structural characteristics and key amino acid residues in the G- and Hsite as well as to provide insights into the mechanism of molecular recognition (Fig. 6). Both enzymes share the same overall fold and domain organization as other plant tau class GSTs (Fig. 6). The RMSD and Qscore between GmGSTU10-10 and GmGSTU4-4-GSH structures were $0.52 \AA$ and 0.92 , respectively, indicating no significant differences in the structure. G-site residues are highly conserved and show no alterations in their positions.

However, significant variations that may contribute to different binding properties were identified in the $\mathrm{H}$-site of the active site, more specifically in helix $\mathrm{H} 9$ at the C-terminal domain (Fig. 7). The C-terminal residues 208-219 at H9 of GmGSTU10-10 and GmGSTU4-4-GSH form a more open conformation as compared to GmGSTU4-4-Nb-GSH. Importantly, helix H9 (residues 202-211) of GmGSTU4-4-Nb-GSH was shown to fold back over the top of the Nterminal domain forming a lid to block the active site pocket in agreement with the induced fit mechanism [16]. A key residue Lys 215 at H9, which acts as a lid over the entrance to the site in GmGSTU4-4-Nb-GSH, is observed to point away from the active site in the GmGSTU10-10 and GmGSTU4-4-GSH structures.

Calculation of the area and volume occupied by each active site using CASTp [51] shows that GmGSTU4-4-GSH and GmGSTU4-4-Nb-GSH have comparable active site pockets ( $453.5 \AA^{2}, 698.3 \AA^{3} ; 483 \AA^{2}, 788.5 \AA^{3}$ ) but significantly larger than that of GmGSTU10-10 (341.9 $\left.\AA^{2}, 531.4 \AA^{3}\right)$, suggesting a difference that may affect GSH and xenobiotic recognition. The smaller size of active site in GmGSTU10-10 may reflect a more compact binding of GSH and probably explains the higher affinity of the enzyme for GSH. Additionally, it is observed that $\mathrm{D}^{103} \mathrm{KKIY}^{107}$ at the upper part of helix H4 (part of the H-site) in GmGSTU4-Nb-GSH forms a sharp kink compared to GmGSTU10-10 and GmGSTU4-4-GSH. This could be explained by the involvement of Tyr107 of GmGSTU4-4 in Nb-GSH binding and the proximity of Tyr107 to the nitrobenzyl moiety of $\mathrm{Nb}$-GSH. In GmGSTU10-10, Tyr107 is replaced by His107, a change that may affect xenobiotic recognition and possibly induce a different extend of conformational changes at the $\mathrm{H}$-site that promote the adaptation of an alternative rate-limiting step (product release) by the enzyme.

\section{Conclusions}

Members of tau GSTs in G. max overlap in their functions, thus providing the plant a broad range of protection. GST function cannot infer from their high sequence similarity to other members of known function. Thus, to understand at molecular level the functional role of GSTs, each member should be characterized individually by biochemical and structural analysis. In the present work, we describe the characterization of a tau class GST isoenzyme from G. max, which is specifically induced following SMV infection. The results showed that GmGSTU10-10 exhibits high antioxidant catalytic function and significantly lower $\mathrm{K}_{\mathrm{m}}$ for GSH compared to other plant GSTs, suggesting that the biological role of the enzyme is to cope with the anti-oxidative metabolism balance at late infection stages. Structural analysis showed that GmGSTU10-10 shares the same overall fold and domain organization of other tau class plant cytosolic GSTs, with major differences in the size of the active site pockets and the structure of the H-site that lead to different kinetic properties and rate-limiting step. Our findings shed light on understanding better the GST structural and catalytic diversity and highlight the pivotal role of GSTs used by plants to cope with biotic stress.

\section{Author contributions}

KS: performed experiments; analyzed data; wrote the paper, AWM: performed experiments; analyzed data; wrote the paper, ACP: planned experiments; wrote the paper, EC: performed experiments; analyzed data, NEL: planned experiments; analyzed data; wrote the paper. 


\section{Acknowledgments}

NEL, ACP, and EC acknowledge financial support from the grant program THALES (grant number 380236), co-funded by the European Union-European Social Fund and National Resources. The grant THALES-"Glutathione transferases: multifunctional molecular tools in red and green biotechnology" falls under the Operational Programme "Education and Lifelong Learning". AWM acknowledges financial support from the Cultural Foundation of Southwestern Finland. ACP thanks Biocenter Finland for infrastructure support and Turku University Foundation (grant number 9237) for financial support. Access to EMBL-Hamburg (c/o DESY) was provided by the European Community's Seventh Framework Programme (FP7/2007-2013) under grant agreements 226716 and 283570 (BioStruct-X project).

\section{References}

[1] E. Chronopoulou, I. Axarli, I. Nianiou-Obeidat, P. Madesis, A. Tsaftaris, N.E. Labrou, Structure and antioxidant catalytic function of plant glutathione transferases, Curr. Chem. Biol. 5 (2011) 64-74

[2] D.P. Dixon, M. Skipsey, R. Edwards, Roles for glutathione transferases in plant secondary metabolism, Phytochemistry 71 (2010) 338-350.

[3] I. Axarli, C. Georgiadou, P. Dhavala, A.C. Papageorgiou, N.E. Labrou, Investigation of the role of conserved residues Ser13, Asn48 and Pro49 in the catalytic mechanism of the tau class glutathione transferase from Glycine max, Biochim. Biophys. Acta 1804 (2010) 662-667.

[4] B. Mannervik, Five decades with glutathione and the GSTome, J. Biol. Chem. 287 (2012) 6072-6083.

[5] K. Benekos, C. Kissoudis, I. Nianiou-Obeidat, N. Labrou, P. Madesis, M. Kalamaki, A. Makris, A. Tsaftaris, Overexpression of a specific soybean GmGSTU4 isoenzyme improves diphenyl ether and chloroacetanilide herbicide tolerance of transgenic tobacco plants, J. Biotechnol. 150 (2010) 195-201.

[6] M. Skipsey, K.M. Knight, M. Brazier-Hicks, D.P. Dixon, P.G. Steel, R. Edwards, Xenobiotic responsiveness of Arabidopsis thaliana to a chemical series derived from a herbicide safener, J. Biol. Chem. 286 (2011) 32268-32276.

[7] S. Govindarajan, B. Mannervik, J.A. Silverman, K. Wright, D. Regitsky, U. Hegazy, T.J. Purcell, M. Welch, J. Minshull, C. Gustafsson, Mapping of amino acid substitutions conferring herbicide resistance in wheat glutathione transferase, ACS Synth. Biol (2014), http://dx.doi.org/10.1021/sb500242x (140619124955009).

[8] R. Thom, I. Cummins, D.P. Dixon, R. Edwards, D.J. Cole, A.J. Lapthorn, Structure of a tau class glutathione S-transferase from wheat active in herbicide detoxification, Biochemistry 41 (2002) 7008-7020.

[9] D.P. Dixon, P.G. Steel, R. Edwards, Roles for glutathione transferases in antioxidant recycling, Plant Signal. Behav. 6 (2011) 1223-1227.

[10] E. Chronopoulou, P. Madesis, B. Asimakopoulou, D. Platis, A. Tsaftaris, N.E. Labrou, Catalytic and structural diversity of the fluazifop-inducible glutathione transferases from Phaseolus vulgaris, Planta 235 (2012) 1253-1269.

[11] E. Chronopoulou, P. Madesis, A. Tsaftaris, N.E. Labrou, Cloning and characterization of a biotic-stress-inducible glutathione transferase from Phaseolus vulgaris, Appl. Biochem. Biotechnol. 172 (2014) 595-609.

[12] I. Cummins, D.J. Wortley, F. Sabbadin, Z. He, C.R. Coxon, H.E. Straker, J.D. Sellars, K. Knight, L. Edwards, D. Hughes, S.S. Kaundun, S.-J. Hutchings, P.G. Steel, R. Edwards, Key role for a glutathione transferase in multiple-herbicide resistance in grass weeds, Proc. Natl. Acad. Sci. U. S. A. 110 (2013) 5812-5817.

[13] S.T. Mashiyama, M.M. Malabanan, E. Akiva, R. Bhosle, M.C. Branch, B. Hillerich, K. Jagessar, J. Kim, Y. Patskovsky, R.D. Seidel, M. Stead, R. Toro, M.W. Vetting, S.C. Almo, R.N. Armstrong, P.C. Babbitt, Large-scale determination of sequence, structure, and function relationships in cytosolic glutathione transferases across the biosphere, PLoS Biol. 12 (2014) e1001843.

[14] A. Moons, Regulatory and functional interactions of plant growth regulators and plant glutathione S-transferases (GSTs), Vitam. Horm. 72 (2005) 155-202.

[15] C. Frova, Glutathione transferases in the genomics era: new insights and perspectives, Biomol. Eng. 23 (2006) 149-169.

[16] I. Axarli, P. Dhavala, A.C. Papageorgiou, N.E. Labrou, Crystallographic and functional characterization of the fluorodifen-inducible glutathione transferase from Glycine max reveals an active site topography suited for diphenylether herbicides and a novel L-site, J. Mol. Biol. 385 (2009) 984-1002.

[17] B. McGonigle, S.J. Keeler, S.M. Lau, M.K. Koeppe, D.P. O'Keefe, A genomics approach to the comprehensive analysis of the glutathione S-transferase gene family in soybean and maize, Plant Physiol. 124 (2000) 1105-1120.

[18] U. Wagner, R. Edwards, D.P. Dixon, F. Mauch, Probing the diversity of the Arabidopsis glutathione S-transferase gene family, Plant Mol. Biol. 49 (2002) 515-532.

[19] P.G. Board, D. Menon, Glutathione transferases, regulators of cellular metabolism and physiology, Biochim. Biophys. Acta Gen. 1830 (2013) 3267-3288.

[20] A. Oakley, Glutathione transferases: a structural perspective, Drug Metab. Rev. 43 (2011) 138-151.

[21] G. Ricci, A.M. Caccuri, M. Lo Bello, N. Rosato, G. Mei, M. Nicotra, E. Chiessi, A.P. Mazzetti, G. Federici, Structural flexibility modulates the activity of human glutathione transferase P1-1: role of helix 2 flexibility in the catalytic mechanism, J. Biol. Chem. 271 (1996) 16187-16192.
[22] M.T. Honaker, M. Acchione, J.P. Sumida, W.M. Atkins, Ensemble perspective for catalytic promiscuity: calorimetric analysis of the active site conformational landscape of a detoxification enzyme, J. Biol. Chem. 286 (2011) 42770-42776.

[23] M. Deponte, Glutathione catalysis and the reaction mechanisms of glutathionedependent enzymes, Biochim. Biophys. Acta 1830 (2013) 3217-3266.

[24] M. Babu, A.G. Gagarinova, J.E. Brandle, A. Wang, Association of the transcriptional response of soybean plants with soybean mosaic virus systemic infection, J. Gen. Virol. 89 (2008) 1069-1080.

[25] M. Arif, S. Hassan, Evaluation of resistance in soybean germplasm to soybean mosaic potyvirus under field conditions, J. Biol. Sci. (2002) 601-604.

[26] T. Shimizu, K. Satoh, S. Kikuchi, T. Omura, The repression of cell wall- and plastidrelated genes and the induction of defense-related genes in rice plants infected with Rice dwarf virus, Mol. Plant Microbe Interact. 20 (2007) 247-254.

[27] A. Morales, J.A. O'Rourke, M. Van, D.E. Mortel, Transcriptome analyses and virus induced gene silencing identify genes in the Rpp4-mediated Asian soybean rust resistance pathway, Funct. Plant Biol. 40 (2013) 1029-1047.

[28] X.-Y. Xu, R. Fan, R. Zheng, C.-M. Li, D.-Y. Yu, Proteomic analysis of seed germination under salt stress in soybeans, J. Zhejiang Univ. Sci. B 12 (2011) 507-517.

[29] Z. Ali, D.Y. Zhang, Z.L. Xu, L. Xu, J.X. Yi, X.L. He, Y.H. Huang, X.Q. Liu, A.A. Khan, R.M Trethowan, H.X. Ma, Uncovering the salt response of soybean by unraveling its wild and cultivated functional genomes using tag sequencing, PLoS ONE 7 (2012) e48819.

[30] N.E. Labrou, L.V. Mello, Y.D. Clonis, Functional and structural roles of the glutathione-binding residues in maize (Zea mays) glutathione S-transferase I, Biochem. J. 358 (2001) 101-110.

[31] D.P. Dixon, A.G. McEwen, A.J. Lapthorn, R. Edwards, Forced evolution of a herbicide detoxifying glutathione transferase, J. Biol. Chem. 278 (2003) 23930-23935.

[32] S.R. Wilson, P.A. Zucker, R.R.C. Huang, A. Spector, Development of synthetic compounds with glutathione peroxidase activity, J. Am. Chem. Soc. 111 (1989) 5936-5939.

[33] H.A. Kramers, Brownian motion in a field of force and the diffusion model of chemical reactions, Physica 7 (1940) 284-304.

[34] M. Jacob, F.X. Schmid, Protein folding as a diffusional process, Biochemistry 38 (1999) 13773-13779.

[35] A.P. Demchenko, O.I. Ruskyn, E.A. Saburova, Kinetics of the lactate dehydrogenase reaction in high-viscosity media, 998 (1989) 196-203.

[36] W.W. Cleland, Statistical analysis of enzyme kinetic data, Methods Enzymol. 63 (1979) 103-138.

[37] J. Oakes, T. Nguyen, B.M. Britt, Arrhenius analysis of the electrophorus electricus acetylcholinesterase-catalyzed hydrolysis of acetylthiocholine, Protein Pept. Lett. 10 (2003) 321-324.

[38] G. Peng, G. Fritzsch, V. Zickermann, H. Schägger, R. Mentele, F. Lottspeich, M. Bostina M. Radermacher, R. Huber, K.O. Stetter, H. Michel, Isolation, characterization and electron microscopic single particle analysis of the NADH:ubiquinone oxidoreductase (complex I) from the hyperthermophilic eubacterium Aquifex aeolicus, Biochemistry 42 (2003) 3032-3039.

[39] Y.X. Fan, P. McPhie, E.W. Miles, Regulation of tryptophan synthase by temperature, monovalent cations, and an allosteric ligand. Evidence from Arrhenius plots, absorption spectra, and primary kinetic isotope effects, Biochemistry 39 (2000) 4692-4703.

[40] W. Kabsch, XDS, Acta Crystallogr. D Biol. Crystallogr. 66 (2010) 125-132.

[41] A.J. McCoy, R.W. Grosse-Kunstleve, P.D. Adams, M.D. Winn, L.C. Storoni, R.J. Read, Phaser crystallographic software, J. Appl. Crystallogr. 40 (2007) 658-674.

[42] M.D. Winn, C.C. Ballard, K.D. Cowtan, E.J. Dodson, P. Emsley, P.R. Evans, R.M. Keegan, E.B. Krissinel, A.G.W. Leslie, A. McCoy, Overview of the CCP4 suite and current developments, Acta Crystallogr. D Biol. Crystallogr. 67 (2011) 235-242.

[43] I. Axarli, P. Dhavala, A.C. Papageorgiou, N.E. Labrou, Crystal structure of Glycine max glutathione transferase in complex with glutathione: investigation of the mechanism operating by the Tau class glutathione transferases, Biochem. J. 422 (2009) 247-256.

[44] P. Emsley, K. Cowtan, Coot: model-building tools for molecular graphics, Acta Crystallogr. D Biol. Crystallogr. 60 (2004) 2126-2132.

[45] P.D. Adams, P.V. Afonine, G. Bunkóczi, V.B. Chen, I.W. Davis, N. Echols, J.J. Headd L.-W. Hung, G.J. Kapral, R.W. Grosse-Kunstleve, A.J. McCoy, N.W. Moriarty, R. Oeffner, R.J. Read, D.C. Richardson, J.S. Richardson, T.C. Terwilliger, P.H. Zwart, PHENIX: a comprehensive Python-based system for macromolecular structure solution, Acta Crystallogr. D Biol. Crystallogr. 66 (2010) 213-221.

[46] S. Lovell, I. Davis, W. Arendall, P. de Bakker, J. Word, M. Prisant, J. Richardson, D. Richardson, Structure validation by Calpha geometry: phi, psi and Cbeta deviation, Proteins 50 (2003) 437-450.

[47] R. Laskowski, M. MacArthur, D. Moss, J. Thornton, PROCHECK: a program to check the stereochemical quality of protein structures, J. Appl. Crystallogr. 26 (1993) 283-291.

[48] E. Potterton, P. Briggs, M. Turkenburg, E. Dodson, A graphical user interface to the CCP4 program suite, Acta Crystallogr. D Biol. Crystallogr. 59 (2003) 1131-1137.

[49] E. Krissinel, K. Henrick, Inference of macromolecular assemblies from crystalline state, J. Mol. Biol. 372 (2007) 774-797.

[50] W. Kabsch, C. Sander, Dictionary of protein secondary structure: pattern recognition of hydrogen-bonded and geometrical features, 22 (1983) 2577-2637.

[51] T.A. Binkowski, S. Naghibzadeh, J. Liang, CASTp: computed atlas of surface topography of proteins, Nucleic Acids Res. 31 (2003) 3352-3355.

[52] J.A. Hernández, M. Rubio, E. Olmos, A. Ros Barceló, P. Martínez Gómez, Oxidative stress induced by long-term plum pox virus infection in peach (Prunus persica) Physiol. Plant. 122 (2004) 486-495.

[53] P. Winayanuwattikun, A.J. Ketterman, An electron-sharing network involved in the catalytic mechanism is functionally conserved in different glutathione transferase classes, J. Biol. Chem. 280 (2005) 31776-31782. 
[54] G. Noctor, G. Queval, A. Mhamdi, S. Chaouch, C.H. Foyer, Glutathione, Arabidopsis Book 9 (2011) e0142:1-32.

[55] I. Sinning, G.J. Kleywegt, S.W. Cowan, P. Reinemer, H.W. Dirr, R. Huber, G.L. Gilliland, R.N. Armstrong, X. Ji, P.G. Board, Structure determination and refinement of human alpha class glutathione transferase A1-1, and a comparison with the Mu and Pi class enzymes, J. Mol. Biol. 232 (1993) 192-212.

[56] L. Prade, R. Huber, B. Bieseler, Structures of herbicides in complex with their detoxifying enzyme glutathione S-transferase-explanations for the selectivity of the enzyme in plants, Structure 6 (1998) 1445-1452.

[57] A. Fersht, Enzyme Structure and Mechanism, W.H. Freeman \& Company, Ltd., New York, 1977.

[58] N.E. Labrou, G.A. Kotzia, Y.D. Clonis, Engineering the xenobiotic substrate specificity of maize glutathione S-transferase I, Protein Eng. Des. Sel. 17 (2004) 741-748.
[59] N. Allocati, E. Casalone, M. Masulli, I. Ceccarelli, E. Carletti, M.W. Parker, C. Di Ilio, Functional analysis of the evolutionarily conserved proline 53 residue in Proteus mirabilis glutathione transferase B1-1, FEBS Lett. 445 (1999) 347-350.

[60] J.R. Prohaska, The glutathione peroxidase activity of glutathione S-transferases, Biochim. Biophys. Acta 611 (1980) 87-98.

[61] J.D. Thompson, D.G. Higgins, T.J. Gibson, CLUSTAL W: improving the sensitivity of progressive multiple sequence alignment through sequence weighting, position-specific gap penalties and weight matrix choice, Nucleic Acids Res. 22 (1994) 4673-4680

[62] R.A. Laskowski, M.B. Swindells, LigPlot +: multiple ligand-protein interaction diagrams for drug discovery, J. Chem. Inf. Model. 51 (2011) 2778-2786.

[63] P. Gouet, E. Courcelle, D.I. Stuart, F. Metoz, ESPript: analysis of multiple sequence alignments in PostScript, 15 (1999) 305-308. 